\title{
ROBERT DALMASSO \\ Un problème de symétrie pour une équation biharmonique
}

\author{
Annales de la faculté des sciences de Toulouse $5^{e}$ série, tome 11, \\ no 3 (1990), p. 45-53 \\ <http://www.numdam.org/item?id=AFST_1990_5_11_3_45_0>
}

(C) Université Paul Sabatier, 1990, tous droits réservés.

L'accès aux archives de la revue «Annales de la faculté des sciences de Toulouse » (http://picard.ups-tlse.fr/ annales/) implique l'accord avec les conditions générales d'utilisation (http://www.numdam.org/conditions). Toute utilisation commerciale ou impression systématique est constitutive d'une infraction pénale. Toute copie ou impression de ce fichier doit contenir la présente mention de copyright.

\section{Numdam}

Article numérisé dans le cadre du programme Numérisation de documents anciens mathématiques http://www.numdam.org/ 


\title{
Un problème de symétrie pour une équation biharmonique
}

\author{
ROBERT DaLMASSO ${ }^{(1)}$
}

RÉSUMÉ. - Nous étudions les solutions positives d'une classe d'équations elliptiques d'ordre quatre vérifiant des conditions aux limites surdéterminées. Nous montrons que l'ouvert borné connexe sur lequel la solution est définie est nécessairement une boule et que la solution est radiale.

Abstract. - We study the positive solutions of some fourth order elliptic equations satisfying over-determined boundary conditions. We prove that the domain on which the solution is defined is necessarily a ball and that the solution is spherically symmetric.

\section{Introduction}

Soit $\Omega \subset \mathbb{R}^{n}$ un ouvert borné connexe de frontière $\partial \Omega$ régulière et soit $u \in C^{4}(\bar{\Omega})$ une solution du problème

$$
\Delta^{2} u=1 \quad \text { dans } \Omega
$$

avec les conditions sur le bord

$$
\begin{cases}u=\frac{\partial u}{\partial \nu}=0 & \text { sur } \partial \Omega \\ \Delta u=c & \text { sur } \partial \Omega\end{cases}
$$

où $c$ désigne une constante et $\partial / \partial \nu$ la dérivée normale extérieure. On a le résultat suivant.

(1) Laboratoire TIM 3 - IMAG, Équipe EDP, Tour IRMA, BP 53 X, 38041 Grenoble Cedex (France) 
THÉORÈME 1.1. - Soit $\Omega \subset \mathbb{R}^{n}$ un ouvert borné connexe avec $\partial \Omega$ de classe $C^{2}$. S'il existe $u \in C^{4}(\bar{\Omega})$ vérifiant (1.1), (1.2) et $u \geq 0$ dans $\Omega$ alors $\Omega$ est une boule et $u$ est radiale.

Remarque 1.1. - Plus précisément $\Omega$ est une boule de rayon $R=$ $(n(n+2) c)^{1 / 2}$ et $u=\left(R^{2}-r^{2}\right)^{2} / 8 n(n+2)$ où $r$ désigne la distance d'un point au centre de la boule.

Remarque 1.2. - J. Serrin [2] a étudié un problème analogue pour l'équation de Poisson.

Remarque 1.3. - Une généralisation pour l'équation $\Delta^{2} u=f(u, \Delta u)$ sera donnée dans la partie 3.

Nous utiliserons à plusieurs reprises le principe du maximum et le principe du maximum au bord que nous rappelons. Soit $D$ un ouvert connexe de $\mathbb{R}^{n}$ et soit $u \in C^{2}(D)$ une fonction vérifiant l'inégalité $\Delta u \geq 0$ dans $D$.

Principe du maximum ([1], p. 15). - Si $u$ atteint un maximum $M$ en un point de $D$ alors $u \equiv M$ dans $D$.

Principe du maximum au bord ([1], p. 33). - Soit $P \in \partial D$ tel que :

a) $u$ est continue au point $P$;

b) $u(x)<u(P)$ pour tout $x \in D$;

c) il existe une boule $B$ dans $D$ avec $P \in \partial B$.

Alors la dérivée normale extérieure au point $P$, si elle existe, vérifie $\partial u / \partial \nu(P)>0$.

\section{Démonstration du théorème 1.1}

Comme dans [2] nous utilisons la technique qui consiste à déplacer des plans perpendiculairement à une direction fixe.

Rappelons ce procédé. Soit $y \in \mathbb{R}^{n}$ un vecteur de norme 1 . Pour $\lambda \in \mathbb{R}$ on définit l'hyperplan $T_{\lambda}$ par l'équation $y \cdot x=\lambda$. Si $\tilde{\lambda}$ est suffisamment grand $T_{\widetilde{\lambda}} \cap \bar{\Omega}=\emptyset$. On déplace alors continûment le plan $T_{\lambda}$ vers $\Omega$ en conservant la même normale (i.e. on diminue $\lambda$ ) jusqu'à ce qu'il rencontre $\bar{\Omega}$. À partir de cet instant on notera $\Sigma(\lambda)$ l'ouvert de $\Omega$ constitué par la partie ouverte de $\Omega$ située du même côté de $T_{\lambda}$ que $T_{\widehat{\lambda}}$ et $\Sigma^{\prime}(\lambda)$ son image dans la symétrie par 
rapport à $T_{\lambda}$. Au début $\Sigma^{\prime}(\lambda)$ sera inclus dans $\Omega$. Si on diminue $\lambda, \Sigma^{\prime}(\lambda)$ va rester dans $\Omega$ jusqu'à ce qu'une des deux situations suivantes se produise :

i) $\Sigma^{\prime}(\lambda)$ devient intérieurement tangent à $\partial \Omega$ en un point $P$ non situé sur $T_{\lambda}$

ii) ou bien $T_{\lambda}$ devient orthogonal à $\partial \Omega$ en un point $Q$.

Nous noterons $T_{\lambda_{1}}$ le plan $T_{\lambda}$ quand il atteint l'une de ces deux positions et $\lambda_{0}=\inf \left\{\lambda \in \mathbb{R} \mid \Omega \cap T_{\lambda}=\emptyset\right\}$. Pour tout $x$ dans $\bar{\Omega}, x^{\lambda}$ désignera le symétrique de $x$ par rapport à $T_{\lambda}$.

Nous allons montrer que $\Omega$ est symétrique par rapport à $T_{\lambda_{1}}$. Admettons pour l'instant ce résultat et démontrons le théorème. Tout d'abord la construction précédente entraîne que $\Omega$ est simplement connexe. Comme la direction y est quelconque on en déduit que $\Omega$ est une boule. Si $R$ désigne le rayon de cette boule et $r$ la distance d'un point au centre de la boule, alors la fonction $\left(R^{2}-r^{2}\right)^{2} / 8 n(n+2)$ est une solution du problème (1.1), (1.2). L'unicité étant immédiate, le théorème est démontré.

Nous aurons besoin des deux lemmes suivants.

Lemme 2.1. - Avec les hypothèses du théorème 1.1, on a $c>0$ (c désignant la constante intervenant dans (1.2)).

Démonstration. - Si $c \leq 0,(1.1)$ et le principe du maximum entraînent $\Delta u \leq 0$ dans $\Omega$ mais alors (1.2) et le principe du maximum au bord entraînent $u=0$ dans $\Omega$ ce qui contredit (1.1).

Lemme 2.2. - Avec les hypothèses du théorème 1.1, il existe $d>0$ tel que $u$ (resp. $\Delta u$ ) est strictement croissante (resp. décroissante) quand on entre dans $\Omega$ dans une direction non tangentielle en restant dans $\Omega$ à une distance inférieure ou égale à $d$ de $\partial \Omega$.

Démonstration. - D'après le principe du maximum au bord on a $\partial \Delta u / \partial \nu(P)>0$, pour tout $P \in \partial \Omega$, d'où le résultat pour $\Delta u$.

Nous allons maintenant montrer que $\partial^{2} u / \partial \nu^{2}(P)=c$ pour tout $P \in \partial \Omega$ et alors la conclusion pour $u$ découle du lemme 2.1. Soit $P \in \partial \Omega$ et considérons un système de coordonnées d'origine $P$ avec l'axe des $x_{n}$ dirigé dans le sens de la normale intérieure en $P$ à $\partial \Omega$. On peut alors représenter localement $\partial \Omega$ par l'équation

$$
\begin{gathered}
x_{n}=\varphi\left(x^{\prime}\right), \quad x^{\prime}=\left(x_{1}, \ldots, x_{n-1}\right) \\
-47-
\end{gathered}
$$


avec $\varphi$ de classe $C^{2}$. On a

$$
\frac{\partial u}{\partial x_{j}}\left(x^{\prime}, \varphi\left(x^{\prime}\right)\right)=0, \quad \text { pour } j=1, \ldots, n .
$$

En dérivant (2.1) par rapport à $x_{k}$ pour $k=1, \ldots, n-1$ on obtient

$$
\frac{\partial^{2} u}{\partial x_{j} \partial x_{k}}\left(x^{\prime}, \varphi\left(x^{\prime}\right)\right)+\frac{\partial^{2} u}{\partial x_{j} \partial x_{n}}\left(x^{\prime}, \varphi\left(x^{\prime}\right)\right) \frac{\partial \varphi}{\partial x_{k}}\left(x^{\prime}\right)=0
$$

pour $1 \leq j \leq n$ et $1 \leq k \leq n-1$. On prend la valeur de (2.2) au point $P$ où $\partial \varphi / \partial x_{k}=0$ et on arrive ̀̀

$$
\frac{\partial^{2} u}{\partial x_{j} \partial x_{k}}(P)=0, \quad \text { pour } j=1, \ldots, n, k=1, \ldots, n-1 .
$$

Or (2.3) entraîne

$$
\frac{\partial^{2} u}{\partial x_{n}^{2}}(P)=(\Delta u)(P)=c .
$$

Ceci achève la démonstration du lemme 2.2.

Pour tout $\lambda \in\left[\lambda_{1}, \lambda_{0}\right.$ [ on définit une fonction $v_{\lambda}$ sur $\Sigma^{\prime}(\lambda)$ en posant

$$
v_{\lambda}(x)=u\left(x^{\lambda}\right) \text { pour } x \in \Sigma^{\prime}(\lambda) .
$$

Il est clair que $v_{\lambda}$ vérifie l'équation

$$
\Delta^{2} v_{\lambda}=1 \text { dans } \Sigma^{\prime}(\lambda)
$$

et les conditions aux limites

$$
\begin{cases}v_{\lambda}=u \text { et } \Delta v_{\lambda}=\Delta u & \text { sur } \partial \Sigma^{\prime}(\lambda) \cap T_{\lambda} \\ v_{\lambda}=0, \frac{\partial v_{\lambda}}{\partial \nu}=0 \text { et } \Delta v_{\lambda}=c & \text { sur } \partial \Sigma^{\prime}(\lambda) \backslash T_{\lambda} .\end{cases}
$$

Comme par construction $\Sigma^{\prime}(\lambda)$ est contenu dans $\Omega$ on peut considérer la fonction $v_{\lambda}-u$ dans $\Sigma^{\prime}(\lambda)$. On a alors

$$
\Delta^{2}\left(v_{\lambda}-u\right)=0 \quad \text { dans } \Sigma^{\prime}(\lambda)
$$

et

$$
\begin{cases}\Delta\left(v_{\lambda}-u\right)=0 & \text { sur } \partial \Sigma^{\prime}(\lambda) \cap T_{\lambda} \\ \Delta\left(v_{\lambda}-u\right) \geq 0 & \text { sur } \partial \Sigma^{\prime}(\lambda) \backslash T_{\lambda}\end{cases}
$$


où la dernière condition résulte du principe du maximum.

Maintenant (2.7), (2.8) et le principe du maximum entraînent

$$
\Delta\left(v_{\lambda}-u\right)>0 \text { dans } \Sigma^{\prime}(\lambda)
$$

ou bien

$$
\Delta\left(v_{\lambda}-u\right)=0 \text { dans } \Sigma^{\prime}(\lambda) .
$$

Si $\lambda \in] \lambda_{1}, \lambda_{0}\left[,(2.10)\right.$ est impossible car $\partial \Sigma^{\prime}(\lambda) \backslash T_{\lambda} \subset \Omega$. D'après le lemme 2.2 , il existe $\eta>0$ tel que pour $\lambda \in] \max \left(\lambda_{1}, \lambda_{0}-\eta\right), \lambda_{0}[$, on a

$$
\begin{cases}u>v_{\lambda} & \text { dans } \Sigma^{\prime}(\lambda) \\ \text { et } & \\ \frac{\partial}{\partial \nu}\left(v_{\lambda}-u\right)(x)>0 & \text { pour tout } x \in T_{\lambda} \cap \Omega,\end{cases}
$$

où la dernière condition résulte du principe du maximum au bord (ici $\nu$ désigne la normale extérieure à $\Sigma^{\prime}(\lambda)$ ). Nous allons montrer que (2.11) est vrai pour tout $\lambda \in] \lambda_{1}, \lambda_{0}$ [. Pour cela on définit $\mu \geq \lambda_{1}$ comme la borne inférieure des $\rho \geq \lambda_{1}$ tels que (2.11) est vrai sur ] $\rho, \lambda_{0}$ [. Il est clair que (2.11) est vrai pour $\lambda \in] \mu, \lambda_{0}\left[\right.$. Montrons que $\mu=\lambda_{1}$. Supposons que $\mu>\lambda_{1}$. Montrons tout d'abord que (2.11) est vrai pour $\lambda=\mu$. Sinon on a

$$
\begin{cases}u \geq v_{\mu} & \text { dans } \Sigma^{\prime}(\mu) \\ \text { et } & \text { tel que } u(x)=v_{\mu}(x) \\ \exists x \in \Sigma^{\prime}(\mu)\end{cases}
$$

ou bien

$$
\begin{cases}u>v_{\mu} & \text { dans } \Sigma^{\prime}(\mu) \\ \text { et } & \text { tel que } \frac{\partial u}{\partial \nu}(x)=\frac{\partial v_{\mu}}{\partial \nu}(x) .\end{cases}
$$

De (2.9) pour $\lambda=\mu$ on déduit que (2.12) est impossible par application du principe du maximum et que (2.13) est impossible par application du principe du maximum au bord. Donc (2.11) est vrai pour $\lambda=\mu$. Par définition de $\mu$ il existe une suite $\left(\mu_{j}\right)_{j \in \mathbb{N}}$ strictement croissante vers $\mu$ (avec $\mu_{j}>\lambda_{1}$ ) et pour chaque $j$ un point $x_{j}$ dans $\Sigma^{\prime}\left(\mu_{j}\right)$ tel que

$$
v_{\mu_{j}}\left(x_{j}\right) \geq u\left(x_{j}\right) .
$$

Une suite extraite de $\left(x_{j}\right)_{j \in \mathbb{N}}$ va converger vers un point $x$ appartenant à $\overline{\Sigma^{\prime}(\mu)}$. On a $v_{\mu}(x) \geq u(x)$. Comme (2.11) est vrai pour $\lambda=\mu$ on en déduit 
que $x$ appartient à $\partial \Sigma^{\prime}(\mu)$ et que $v_{\mu}(x)=u(x)$. En utilisant le lemme 2.2 et (2.11) pour $\lambda=\mu$, on montre facilement que $x$ ne peut pas appartenir à $T_{\mu}$. Donc $x \in \partial \Sigma^{\prime}(\mu) \backslash T_{\mu} \subset \Omega$. Le principe du maximum au bord entraîne que $\partial\left(v_{\mu}-u\right) / \partial \nu(x)>0$. D'après $(1.2)$ on a $v_{\mu}(x)=0$ et $\partial v_{\mu} / \partial \nu(x)=0$ donc $u(x)=0$ et $\partial u / \partial \nu(x)<0$, mais ceci contredit le fait que $u \geq 0$ dans $\Omega$. Ainsi, notre assertion est démontrée.

Par continuité, on a donc

$$
u \geq v_{\lambda_{1}} \text { dans } \Sigma^{\prime}\left(\lambda_{1}\right)
$$

Si pour $\lambda=\lambda_{1}$ on est dans la situation (2.10) alors $\Delta u=c \operatorname{sur} \partial \Sigma^{\prime}\left(\lambda_{1}\right) \backslash T_{\lambda_{1}}$ et ceci entraîne que $\Sigma^{\prime}\left(\lambda_{1}\right)$ coïncide avec la partie de $\Omega$ située du même côté de $T_{\lambda_{1}}$ que $\Sigma^{\prime}\left(\lambda_{1}\right)$, c'est-à-dire que $\Omega$ est symétrique par rapport à $T_{\lambda_{1}}$. Pour achever la démonstration du théorème il suffit de montrer que (2.9) est impossible pour $\lambda=\lambda_{1}$. Si on est dans le cas i), c'est-à-dire $\Sigma^{\prime}\left(\lambda_{1}\right)$ est intérieurement tangent à $\partial \Omega$ en un point $P$ non situé sur $T_{\lambda_{1}}$ : comme $u(P)=v_{\lambda_{1}}(P),(2.9)$ pour $\lambda=\lambda_{1},(2.14)$ et le principe du maximum au bord entraînent

$$
\frac{\partial}{\partial \nu}\left(v_{\lambda_{1}}-u\right)(P)>0
$$

mais ceci contredit le fait que

$$
\frac{\partial u}{\partial \nu}(P)=\frac{\partial v_{\lambda_{1}}}{\partial \nu}(P)=0
$$

Donc (2.9) pour $\lambda=\lambda_{1}$ est impossible dans le cas i).

Dans le cas ii), $Q$ est un angle droit de $\Sigma^{\prime}\left(\lambda_{1}\right)$ et on ne peut donc pas utiliser le principe du maximum au bord. Comme dans [2] nous allons montrer que $v_{\lambda_{1}}-u$ a un zéro d'ordre deux au point $Q$ et ensuite on obtient une contradiction en utilisant le lemme 1 de [2] que nous rappelons.

Lemme 1. [2]. - Soit $D^{\prime}$ un ouvert borné connexe de $\mathbb{R}^{n}$ avec $\partial D^{\prime}$ de classe $C^{2}$ et soit $T$ un plan contenant la normale $\dot{a} \partial D^{\prime}$ en un point $Q$. Notons $D$ l'une des deux parties ouvertes de $D^{\prime}$ délimitées par $T$ et supposons qu'il existe $w \in C^{2}(\bar{D})$ vérifiant $\Delta u \geq 0$ dans $D, w \leq 0$ dans $D$ et $w(Q)=0$. Si s est une direction en $Q$ entrant dans $D$ de façon non tangentielle alors

$$
\frac{\partial w}{\partial s}(Q)<0 \quad \text { ou } \quad \frac{\partial^{2} w}{\partial s^{2}}(Q)<0
$$


$\grave{a}$ moins que $w=0$ dans $D$.

Montrons donc que $v_{\lambda_{1}}-u$ a un zéro d'ordre deux en $Q$. Considérons un système de coordonnées d'origine $Q$ avec l'axe des $x_{n}$ dirigé dans le sens de la normale intérieure en $Q$ à $\partial \Omega$ et l'axe des $x_{1}$ normal à $T_{\lambda_{1}}$. On est donc dans les mêmes conditions que dans la démonstration du lemme 2.2 dont nous reprenons les notations en remplaçant $P$ par $Q .(2.5)$ entraîne

$$
v_{\lambda_{1}}\left(x_{1}, x_{2}, \ldots, x_{n}\right)=u\left(-x_{1}, x_{2}, \ldots, x_{n}\right) \text {. }
$$

(2.3) et (2.4) entraînent alors que les dérivées secondes de $u$ et de $v_{\lambda_{1}}$ coïncident au point $Q$. Compte tenu de (1.2), $v_{\lambda_{1}}-u$ a bien un zéro d'ordre deux au point $Q$. Ceci achève la démonstration du théorème 1.1.

\section{Extension du théorème 1.1 \\ à une équation plus générale}

Dans cette partie nous allons démontrer le résultat suivant.

THÉORÈmE 3.1. - Soit $\Omega \subset \mathbb{R}^{n}$ un ouvert borné connexe avec $\partial \Omega$ de classe $C^{2}$. Soit $f: \mathbb{R}^{2} \rightarrow(0,+\infty)$ une fonction vérifiant les conditions suivantes :

$$
\begin{aligned}
& \text { pour tout } y \in \mathbb{R}, x \rightarrow f(x, y) \text { est croissante, } \\
& \text { pour tout } x \in \mathbb{R}, y \rightarrow f(x, y) \text { est décroissante. }
\end{aligned}
$$

Supposons qu'il existe une fonction $u \geq 0$ dans $C^{4}(\bar{\Omega})$ vérifiant l'équation

$$
\Delta^{2} u=f(u, \Delta u) \quad \text { dans } \Omega
$$

avec les conditions sur le bord

$$
\begin{cases}u=\frac{\partial u}{\partial \nu}=0 & \text { sur } \partial \Omega, \\ \Delta u=c & \text { sur } \partial \Omega,\end{cases}
$$

où c désigne une constante.

Alors $\Omega$ est une boule et $u$ est radiale.

Démonstration. - Tout d'abord il est clair que les lemmes 2.1 et 2.2 sont encore vrais. La technique utilisée ici étant la même que celle utilisée pour 
démontrer le théorème 1.1 , nous conservons les notations introduites dans la partie 2. On définit encore $v_{\lambda}$ par (2.5) pour $\lambda \in\left[\lambda_{1}, \lambda_{0}\right.$ [ et $v_{\lambda}$ vérifie l'équation

$$
\Delta^{2} v_{\lambda}=f\left(v_{\lambda}, \Delta v_{\lambda}\right) \text { dans } \Sigma^{\prime}(\lambda)
$$

avec les conditions aux limites (2.6). D'après le lemme 2.2, il existe $\eta>0$ tel que pour $\lambda \in] \max \left(\lambda_{1}, \lambda_{0}-\eta\right), \lambda_{0}[$, on a

$$
\left\{\begin{array}{l}
\Delta\left(v_{\lambda}-u\right)>0 \text { dans } \Sigma^{\prime}(\lambda) \\
\text { et } \\
v_{\lambda}-u<0 \quad \text { dans } \Sigma^{\prime}(\lambda) .
\end{array}\right.
$$

Nous allons montrer que (3.5) est vrai pour tout $\lambda \in] \lambda_{1}, \lambda_{0}$ [. Pour cela on définit $\mu \geq \lambda_{1}$ comme la borne inférieure des $\rho \geq \lambda_{1}$ tels que (3.5) est vrai sur $] \rho, \lambda_{0}[$. Il est clair que (3.5) est vrai pour $\lambda \in] \mu, \lambda_{0}[$. Montrons que $\mu=\lambda_{1}$. Supposons que $\mu>\lambda_{1}$. Montrons tout d'abord que (3.5) est vrai pour $\lambda=\mu$. Sinon on a

$$
\begin{cases}\Delta\left(v_{\mu}-u\right) \geq 0 & \text { dans } \Sigma^{\prime}(\mu) \\ \text { et } & \text { dans } \Sigma^{\prime}(\mu)\end{cases}
$$

(il y a a priori deux autres cas mais le principe du maximum montre que l'un ne peut pas se produire et que l'autre se ramène à la situation (3.6)). Or (3.1) et (3.2) entraînent que $\Delta^{2}\left(v_{\mu}-u\right) \leq 0$ dans $\Sigma^{\prime}(\mu)$ et donc, par le principe du maximum, on a $\Delta\left(v_{\mu}-u\right)>0$ dans $\Sigma^{\prime}(\mu)$ ou bien $\Delta\left(v_{\mu}-u\right)=0$ dans $\Sigma^{\prime}(\mu)$. Mais ce dernier cas ne peut pas se produire car $\Delta v_{\mu}=c>\Delta u$ sur $\partial \Sigma^{\prime}(\mu) \backslash T_{\mu}$. Donc (3.5) est vrai pour $\lambda=\mu$.

Par définition de $\mu$ il existe une suite $\left(\mu_{j}\right)_{j \in \mathbb{N}}$ strictement croissante vers $\mu\left(\right.$ avec $\mu_{j}>\lambda_{1}$ ) et pour chaque $j$ un point $x_{j}$ dans $\Sigma^{\prime}\left(\mu_{j}\right)$ tel que

$$
v_{\mu_{j}}\left(x_{j}\right) \geq u\left(x_{j}\right)
$$

ou un point $y_{j}$ dans $\Sigma^{\prime}\left(\mu_{j}\right)$ tel que

$$
\left(\Delta v_{\mu_{j}}\right)\left(y_{j}\right) \leq(\Delta u)\left(y_{j}\right) .
$$

Si on est dans la situation (3.7), on fait le même raisonnement que dans la démonstration du théorème 1.1. Examinons la situation (3.8). Une suite extraite de $\left(y_{j}\right)_{j \in \mathbb{N}}$ va converger vers un point $y$ appartenant à $\overline{\Sigma^{\prime}(\mu)}$. 
On a donc $\left(\Delta v_{\mu}\right)(y) \leq(\Delta u)(y)$. Comme (3.5) est vrai pour $\lambda=\mu$, on en déduit que $y$ appartient à $\partial \Sigma^{\prime}(\mu)$ et que $\left(\Delta v_{\mu}\right)(y)=(\Delta u)(y)$. Si $y \in \partial \Sigma^{\prime}(\mu) \backslash T_{\mu} \subset \Omega$ le principe du maximum entraîne que $c=\left(\Delta v_{\mu}\right)(y)>$ $(\Delta u)(y)$, ce qui est impossible. Enfin, en utilisant le lemme 2.2, (3.5) pour $\lambda=\mu$ et le principe du maximum au bord, on montre facilement que $y$ ne peut pas appartenir à $T_{\mu}$. Ainsi notre assertion est démontrée.

Par continuité on a

$$
\left\{\begin{array}{l}
\Delta\left(v_{\lambda_{1}}-u\right) \geq 0 \quad \text { dans } \Sigma^{\prime}\left(\lambda_{1}\right) \\
\text { et } \\
v_{\lambda_{1}}-u \leq 0 \quad \text { dans } \Sigma^{\prime}\left(\lambda_{1}\right) .
\end{array}\right.
$$

Comme précédemment, on montre que $\Delta\left(v_{\lambda_{1}}-u\right)>0$ dans $\Sigma^{\prime}\left(\lambda_{1}\right)$ ou bien $\Delta\left(v_{\lambda_{1}}-u\right)=0$ dans $\Sigma^{\prime}\left(\lambda_{1}\right)$ et on achève la démonstration du théorème 3.1 comme dans la partie 2 .

\section{Bibliographie}

[1] Gilbarg (D.) and Trudinger (N.S.) - Elliptic partial differential equations of second order,

Springer Verlag, vol. 224, Berlin-Heidelberg-New York (1977).

[2] SERRIN (J.) . - A symmetry problem in potential theory, Arch. Ration. Mech., vol. 43 (1971) pp. 304-318. 